\title{
Aneurysmal Bone Cyst
}

National Cancer Institute

\section{Source}

National Cancer Institute. Aneurysmal Bone Cyst. NCI Thesaurus. Code C3516.

A locally aggressive and destructive benign cystic lesion of the bone. It is characterized by the formation of multiloculated hemorrhagic cystic spaces which are separated by fibrous septa. It can arise from any bone, but usually affects the metaphysis of long bones. It manifests with pain and swelling and may recur following curettage. 